\title{
The O-type eclipsing contact binary LY Aurigae - member of a quadruple system ${ }^{\star}$
}

\author{
Pavel Mayer ${ }^{1}$, Horst Drechsel ${ }^{2}$, Petr Harmanec ${ }^{1}$, Stephenson Yang ${ }^{3}$, and Miroslav Šlechta ${ }^{4}$ \\ 1 Astronomical Institute of Charles University, Faculty of Mathematics and Physics, V Holešovičkách 2, 18000 Praha 8, \\ Czech Republic \\ e-mail: mayer@cesnet.cz \\ 2 Dr Karl Remeis Observatory \& ECAP, Astronomical Institute, Friedrich-Alexander University Erlangen-Nuremberg, \\ Sternwartstr. 7, 96094 Bamberg, Germany \\ 3 Physics \& Astronomy Department, University of Victoria, PO Box 3055 STN CSC, Victoria, BC V8W 3P6, Canada \\ ${ }^{4}$ Astronomical Institute, Academy of Sciences of the Czech Republic, 25165 Ondřejov, Czech Republic
}

Received 27 June 2013 / Accepted 25 August 2013

\begin{abstract}
The eclipsing binary LY Aur (O9 II + O9 III) belongs to the rare class of early-type contact systems. We obtained 23 new spectra at the Ondřejov and Dominion Astrophysical Observatories, which were analysed with four older Calar Alto and one ELODIE archive spectra. A new result of this study is that the visual companion of LY Aur - the spectral lines of which are clearly seen in our spectra - is also an SB1 binary having an orbital period of 20d46, an eccentric orbit, and a radial velocity semi-amplitude of $33 \mathrm{~km} \mathrm{~s}^{-1}$. The $\mathrm{H} \alpha$ line blend contains an emission component, which shows dependence on the orbital phase of the eclipsing system, with the strongest emission around the secondary eclipse. Revised elements of the eclipsing binary and the orbital solution of the companion binary are determined from our set of spectra and new light-curve solutions of the eclipsing pair. The mass of the primary of $25.5 M_{\odot}$ agrees well with its spectral type, whereas the secondary mass of $14 M_{\odot}$ is smaller than expected. From an $\mathrm{O}-\mathrm{C}$ analysis of the minimum times of LY Aur that span more than 40 years, we found that the orbital period is decreasing, indicating the presence of interaction processes. The system is likely in a phase of non-conservative mass exchange.
\end{abstract}

Key words. stars: early-type - binaries: eclipsing - binaries: visual - stars: individual: LY Aurigae

\section{Introduction}

There are only several galactic O-type eclipsing binaries where at least one component has already evolved away of the main sequence. These rapid phases of evolution are particularly suitable to the tests of modern stellar-evolution models. Each of these binaries is unique, but one characteristic property they share is that they exhibit a variable $\mathrm{H} \alpha$ emission in their spectra. Examples of these systems are V1007 Sco, $\delta$ Ori, and several binaries containing supergiants. All these binaries follow individual ways of evolution; the details of which are not yet fully understood. The object of this study, LY Aur, might be a good test case, being easier to study due to its deep eclipses and only modest emission. However, as we shall show in this paper, it is not a simple object since it is a member of a quadruple system. This, however, can make its history even more interesting to study.

The object LY Aur (HD 35921; ADS 4072A) was discovered as an eclipsing binary by Mayer (1968). Sota et al. (2011) classified the binary as O9.5 II + O9 III. Although the system is fairly bright and an important and conspicuous early-type binary, it is surprising that there were only few attempts to determine accurate system properties. The reported results are even partly inconsistent. One possible reason might be attributed to the orbital period that is very close to four days. A near-integer

* Based on spectral observations collected at the German-Spanish Observatory, Calar Alto, Spain; Dominion Astrophysical Observatory, Canada; Ondřejov Observatory, Czech Republic, and an archival Haute Provence Observatory ELODIE spectrum. period value often presents a problem when the light- or radialvelocity (RV) curves are to be obtained from a single location. Since LY Aur has a visual companion that is only 0.6 apart, a solution of the light curve is hampered by the uncertain contribution of light from this third component. The companion is also responsible for uncertainties in the mass ratio of the binary. The presence of some emission in the $\mathrm{H} \alpha$ line was already noted by Thaller \& Gies (1996), and Thaller (1997) presented an H $\alpha$ profile with a modest emission at the red wing of the line. LY Aur is also included in the list of emission-line objects by Kohoutek \& Wehmeyer (1997). See Sect. 3 for more details on the H $\alpha$ emission in our spectra.

The extended set of spectra made it possible to obtain more reliable values for the contribution of the third body and for the mass ratio of the eclipsing pair. The results of our analysis of the "observed minus calculated" times of minima $(\mathrm{O}-\mathrm{C})$ are described in Sect. 2. In Sects. 3 and 4, spectroscopic results for the eclipsing pair are presented, while Sect. 5 deals with the lightcurve solution and the binary parameters. In Sect. 6, the third component is described. Section 7 deals with the spectra disentangling and the results are discussed in Sect. 8.

\section{Ephemeris}

The published minima of LY Aur are collected in Table 1 (except those with large errors) and plotted against the epoch of the eclipsing binary orbit in Fig. 1. 


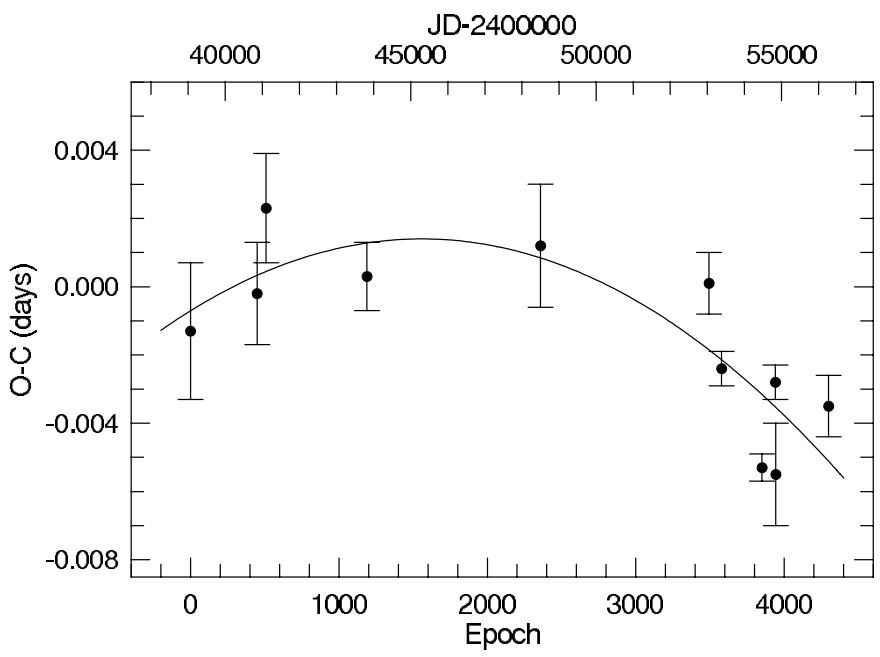

Fig. 1. O-C diagram of the times of minima of LY Aur during more than 40 years; the general trend indicates a decreasing period.

Table 1. Published times of minima for LY Aur.

\begin{tabular}{lcccc}
\hline \hline $\begin{array}{l}\text { HJD } \\
-2400000\end{array}$ & $\begin{array}{c}\text { m.e. } \\
\text { day }\end{array}$ & Epoch & $\begin{array}{c}\text { O-C } \\
\text { day }\end{array}$ & Source \\
\hline 39061.4630 & 20 & 0.0 & -0.0016 & 1 \\
40858.5835 & 15 & 449.0 & -0.0005 & 2 \\
41102.7381 & 16 & 510.0 & 0.0020 & 3 \\
43820.4290 & 10 & 1189.0 & -0.0000 & 4 \\
48503.347 & 18 & 2359.0 & 0.0012 & 5 \\
53046.1756 & 9 & 3494.0 & -0.0002 & 6 \\
53382.3826 & 5 & 3578.0 & -0.0024 & 7 \\
54471.0581 & 4 & 3850.0 & -0.0053 & 8 \\
54833.2860 & 5 & 3940.5 & -0.0028 & 9 \\
54847.2920 & 15 & 3944.0 & -0.0058 & 10 \\
56268.1794 & 9 & 4299.0 & -0.0035 & 8 \\
\hline
\end{tabular}

References. 1 - Mayer (1968); 2 - Mayer \& Horák (1971); 3 - Mayer (1980, meas. at Hvar); 4 - Mayer (1980, normal min. of Hall \& Heiser 1972, and Landolt \& Blondeau 1972); 5 - HIPPARCos, Perryman \& ESA (1997); 6 - Krajci (2005); 7 - Mayer et al. (2006); 8 - Zhao \& Zhu (priv. comm.); 9 - Witt (priv. comm.); 10 - Hubscher et al. (2010).

In what follows we use the ephemeris published by Mayer et al. (2006):

Pri.Min. = HJD $2439061.4646+4.0024932 \times$ E.

By now, the period was considered constant, but it is obvious that the period is decreasing as it follows from the recently measured times of minima (e.g. Hubscher et al. 2010). The curve in Fig. 1 is drawn according to the quadratic ephemeris:

Pri.Min. = HJD $2439061.4639+4.0024959 \times E-0.87 \times 10^{-9} \times E^{2}$.

However, the linear ephemeris (1) suits sufficiently well for the purpose of this paper.

\section{Spectroscopic observations and results}

Besides the early RVs by Mayer (1968), RVs were also measured by Andersen et al. (1974, hereafter ABH) on photographic spectra; they gave the ratio of the secondary and primary masses as $q=m_{2} / m_{1}=0.375$. Stickland et al. (1994) measured IUE spectra with the result $q=0.56$. Popper (1982) wrote that his study based on the Lick Observatory spectra fully confirmed the results of $\mathrm{ABH}$ but he interpreted the mass ratio differently $(q=0.66)$ recognizing the blending of the primary and the third body lines. However, no modern (CCD) spectroscopy was reported so far.

The spectra used in the following are listed in Table 2. Four spectra (CA) were obtained at the Calar Alto Observatory with the coudé spectrograph at the $2.2 \mathrm{~m}$ telescope in the year 1993 (dispersion $8.5 \AA \mathrm{mm}^{-1}$, resolving power 30000 ); one was retrieved from the ELODIE archive (Moultaka et al. 2004). Three spectra (DAO) were exposed with the coudé spectrograph at the $1.22 \mathrm{~m}$ telescope of the Dominion Astrophysical Observatory with a dispersion of $10 \AA \mathrm{mm}^{-1}$ and a resolving power of 21700 . The remaining 20 spectra were obtained with the coudé spectrograph at the $2 \mathrm{~m}$ telescope of the Ondřejov Observatory (dispersion $17.2 \AA \mathrm{mm}^{-1}$, resolving power 12700 ).

The strength of $\mathrm{H} \alpha$ emission is roughly estimated (on a relative scale between 0 and 5) and given in Table 2. Among our 26 spectra of the $\mathrm{H} \alpha$ region, two exhibit strong emission features (see Fig. 4), and several others show at least a modest one. The strongest emission was observed at phases close to the secondary minimum; here, of course, the emission is enhanced due to the fainter continuum. In other cases, a marginal red-shifted emission similar to that shown by Thaller (1997) for LY Aur is evident. A weak emission also appears several times on the blue wing of the line. Some emission might be present in the rest of the spectra but is so weak that it is not rising above the continuum. However, the $\mathrm{H} \alpha$ profiles are strange in the sense that the primary component more or less corresponds to expectation, while the secondary component is weaker than would accord to its spectral type: the average effective widths (EWs) around phases 0.25 and 0.75 are 0.85 (primary) and 0.36 (secondary), respectively, although they should be $>1$ for both stars. A possible explanation of this discrepancy might be a partial filling of the absorption components by emission. This behaviour was also noted by Thaller \& Gies (1996) for LY Aur, who proposed an investigation of the phase dependence on the strength of the line profiles in the O-type SB2 systems.

According to the published light-curve solutions by Li \& Leung (1985) or by Drechsel et al. (1989, hereafter DLM), the temperature of the secondary component $T_{\text {eff2 }}$ is about $3100 \mathrm{~K}$ lower than $T_{\text {eff } 1}$. If we adopt instead the classification by Sota et al. (2011) and the calibration by Martins et al. (2005), $T_{\text {eff2 }}$ is more than $1000 \mathrm{~K}$ higher than $T_{\text {eff1 }}$ (no matter whether the observational or theoretical temperature scale of Martins et al. 2005 is used). In Fig. 2, we show the profiles of He I 4471 and the He II 5411 and 4686 lines in the ELODIE spectrum. In Table 3, the equivalent widths (measured in the ELODIE spectrum) are listed. The He I/He II ratio depends on the temperature, and according to our EW measurements, the temperature difference of the eclipsing binary components is not so high, as suggested by the spectral classification of Sota et al. (2011). For instance, we found $\log E W_{4471}-\log E W_{4541}=0.35$ for the primary component, which corresponds to the spectral type O9 according to Conti \& Alschuler (1971). If the classification of O9 II+O9 III is right, $T_{\text {eff2 }}$ should be only 100 to $200 \mathrm{~K}$ higher than $T_{\text {eff1 }}$. We therefore attempt to explain this difference by a new light-curve solution (Sect. 6).

\section{Radial velocities of the eclipsing binary}

The RVs, as measured for the He I 6678 line using a Gaussian fitting, are given in Table 2. Examples of the profiles of this line are shown in Fig. 9. The variable continuum contributions 
Table 2. Spectra of LY Aur.

\begin{tabular}{|c|c|c|c|c|c|c|c|c|c|}
\hline $\begin{array}{l}\text { HJD } \\
-2400000\end{array}$ & $\begin{array}{c}\text { Exp. } \\
\text { s }\end{array}$ & $\begin{array}{l}\text { Region } \\
\mathrm{nm}\end{array}$ & $\begin{array}{l}\text { Phase } \\
\text { LY Aur }\end{array}$ & $\begin{array}{l}\text { RV Pri. } \\
\mathrm{km} \mathrm{s}^{-1}\end{array}$ & $\begin{array}{c}\mathrm{RV} \mathrm{Sec}^{a}{ }^{a} \\
\mathrm{~km} \mathrm{~s}^{-1}\end{array}$ & $\begin{array}{l}\text { Emission } \\
\text { strength }^{b}\end{array}$ & $\begin{array}{l}\text { Phase } \\
\text { vis. comp. }\end{array}$ & $\begin{array}{l}\text { Vis. comp. } \\
\mathrm{RV} \mathrm{km} \mathrm{s}^{-1}\end{array}$ & Source \\
\hline 48999.5542 & 2100 & $482-503$ & 0.9748 & & & & 0.5535 & 9 & CA \\
\hline 49000.5424 & 1800 & $651-672$ & 0.2217 & -159 & 288 & 1 & 0.6018 & 5 & CA \\
\hline 49001.5799 & 2000 & $651-672$ & 0.4809 & 6 & & 2 & 0.6525 & -4 & $\mathrm{CA}$ \\
\hline 49002.5604 & 1800 & $651-672$ & 0.7258 & 168 & -287 & $1 b^{c}$ & 0.7004 & -9 & CA \\
\hline 53331.5784 & 2700 & $400-680$ & 0.3062 & -162 & 270 & 0 & 0.2414 & 25 & ELODIE \\
\hline 53387.2875 & 1899 & $626-677$ & 0.2248 & -132 & 295 & 1 & 0.9637 & 30 & Ond. \\
\hline 55060.5720 & 2000 & $625-676$ & 0.2853 & -160 & 280 & 2 & 0.7301 & -14 & Ond. \\
\hline 55108.4858 & 2800 & $625-676$ & 0.2563 & -149 & 283 & 2 & 0.0715 & -1 & Ond. \\
\hline 55108.5320 & 2400 & $532-583$ & 0.2679 & & & & & & Ond. \\
\hline 55250.3571 & 1400 & $626-677$ & 0.7021 & 168 & -287 & 1 & 0.0041 & -27 & Ond. \\
\hline 55266.2872 & 2100 & $626-677$ & 0.6821 & 166 & -287 & 0 & 0.7826 & -25 & Ond. \\
\hline 55279.3606 & 2130 & $626-677$ & 0.9484 & 43 & -135 & $1 b^{c}$ & 0.4214 & 23 & Ond. \\
\hline 55501.5024 & 2200 & $626-677$ & 0.4493 & -25 & 173 & 5 & 0.2767 & 28 & Ond. \\
\hline 55578.3032 & 1700 & $625-676$ & 0.6375 & 124 & -288 & 0 & 0.0295 & -25 & Ond. \\
\hline 55602.5532 & 1629 & $625-676$ & 0.6962 & 151 & -274 & 0 & 0.2145 & 21 & Ond. \\
\hline 55616.4854 & 4013 & $625-676$ & 0.1771 & -138 & 276 & 0 & 0.8953 & -38 & Ond. \\
\hline 56330.4824 & 2200 & $625-676$ & 0.5652 & 36 & -189 & 3 & 0.7854 & -27 & Ond. \\
\hline 56354.4419 & 4380 & $625-675$ & 0.5513 & 30 & -172 & 5 & 0.9561 & -37 & Ond. \\
\hline 56357.4800 & 2836 & $625-675$ & 0.3105 & -139 & 271 & 1 & 0.1046 & 9 & Ond. \\
\hline 56381.6619 & 2000 & $632-692$ & 0.3521 & -124 & 228 & 2 & 0.2863 & 29 & DAO \\
\hline 56382.6698 & 2201 & $632-692$ & 0.6039 & 84 & -187 & 0 & 0.3355 & 30 & DAO \\
\hline 56383.6685 & 1600 & $632-692$ & 0.8534 & 116 & -249 & 0 & 0.3843 & 24 & DAO \\
\hline 56390.4108 & 2823 & $625-675$ & 0.5379 & 27 & & 2 & 0.7138 & -12 & Ond. \\
\hline 56396.4057 & 1894 & $625-676$ & 0.0357 & & 50 & $1 b^{c}$ & 0.0068 & -28 & Ond. \\
\hline 56400.2891 & 1118 & $625-676$ & 0.0060 & & 17 & $1 b^{c}$ & 0.1965 & 21 & Ond. \\
\hline 56406.3318 & 4090 & $625-676$ & 0.5157 & 17 & & 3 & 0.4918 & 14 & Ond. \\
\hline 56407.2943 & 847 & $625-676$ & 0.7562 & 160 & -296 & 1 & 0.5388 & 9 & Ond. \\
\hline 56421.3195 & 1241 & $625-676$ & 0.2603 & -151 & 282 & 1 & 0.2242 & 24 & Ond. \\
\hline
\end{tabular}

Notes. ${ }^{(a)}$ At phases close to 0.5 , the secondary lines are absent. ${ }^{(b)}$ Relative intensity scale from 0 to 5. (c) "b" means that the emission occurs at the blue wing of the profile.

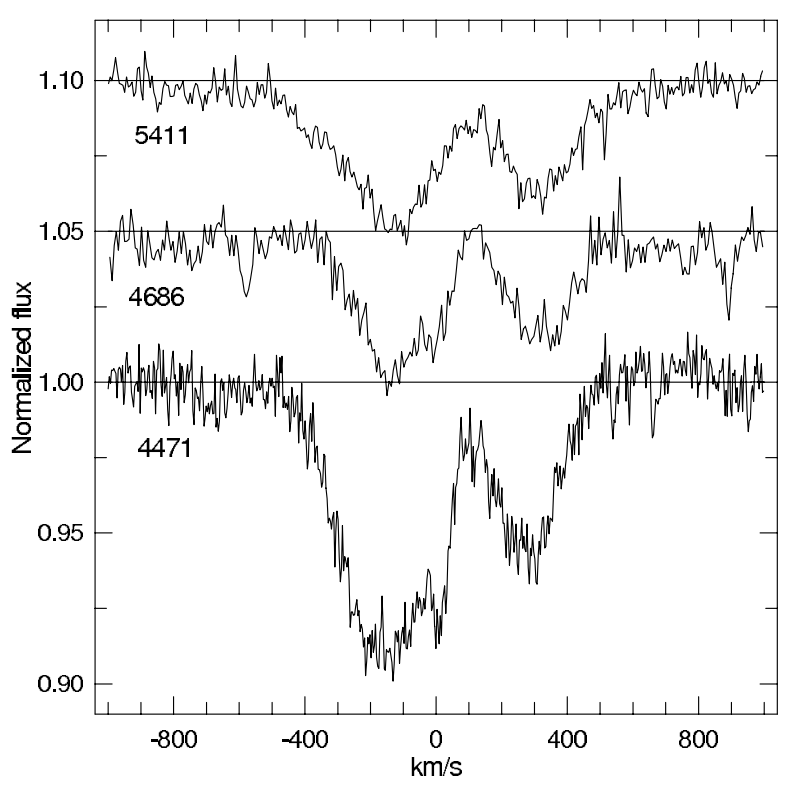

Fig. 2. Profiles of He I 4471, He II 4686, and He II 5411 lines in the ELODIE spectrum (phase 0.306). The narrow absorption close to zero velocity in the line He I 4471 is the line of the visual component; absorptions at -580 and $+900 \mathrm{~km} \mathrm{~s}^{-1}$ in He II 4686 belong to the visual component (lines O II 4676 and 4699).

of individual system components to the integral light, as they change according to the phase of the eclipsing binary, were
Table 3. Equivalent widths ( $\mathrm{A})$ of several lines of the eclipsing binary components in the ELODIE spectrum.

\begin{tabular}{lccc}
\hline \hline Line & Primary & Secondary & Theory $^{a}$ \\
\hline $\mathrm{H} \alpha$ & 0.85 & 0.36 & 2.55 \\
$\mathrm{H} \beta$ & 0.99 & 0.54 & 2.34 \\
$\mathrm{He}$ I 4471 & 0.40 & 0.18 & 0.55 \\
$\mathrm{He}$ I 4922 & 0.23 & 0.17 & 0.35 \\
$\mathrm{He}$. 6678 & 0.39 & 0.25 & 0.44 \\
$\mathrm{He}$ II 4541 & 0.18 & 0.09 & 0.21 \\
$\mathrm{He}$ II 5411 & 0.25 & 0.17 & 0.41 \\
\hline
\end{tabular}

Notes. ${ }^{(a)}$ Calculated from synthetic spectra of Lanz \& Hubeny (2003) for $T_{\text {eff }}=31000 \mathrm{~K}$ and $\log g=3.50$.

accounted for when measuring the RVs. However, the following problems remain:

- Although the secondary lines are well represented by Gaussian profiles, the primary lines are not; they are wider with a flat bottom. We approximated them by a symmetric combination of three Gaussian profiles.

- The He II lines of the primary are not symmetrical; see Fig. 2. This effect is probably the result of an asymmetric temperature distribution over the stellar surface. In the given temperature range, the temperature dependence of the neutral helium lines is much weaker than that of the ionized helium lines. 

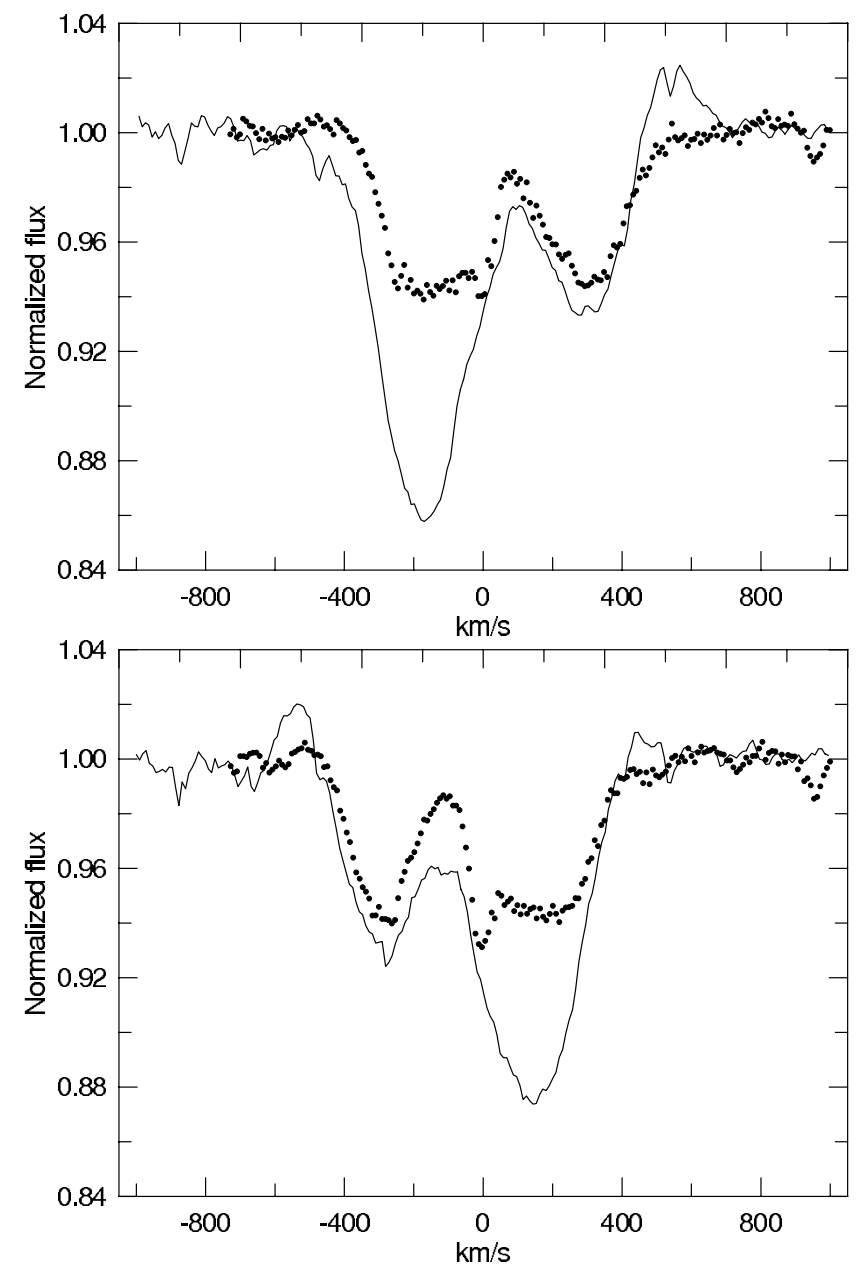

Fig. 3. Top: primary (left) and secondary (right) $\mathrm{H} \alpha$ lines at phase 0.222 . For comparison, the He I 6678 line is added (dots). Bottom: primary (right) and secondary (left) $\mathrm{H} \alpha$ lines at phase 0.726 . For comparison, the He I 6678 line is added (dots).

The primary and secondary RVs, according to the measurements of the He I 6678, line are plotted against orbital phase in Fig. 5. These are the first RV curves based on the ground-based RVs. They confine the true mass ratio to a value close to $q=0.55$. The theoretical RV curves plotted in Fig. 4 are calculated using the Wilson-Devinney (WD) code (Wilson \& Devinney 1971) with the elements based on the new photometric solution (cf. Sect. 6) and on the spectroscopic values of $K_{1}, K_{2}$, and $V \gamma$ obtained in this study.

\section{Spectra disentangling}

To elaborate on the results discussed above, we used the program KOREL ${ }^{1}$ developed by Hadrava (2004) for spectra disentangling in the Fourier space. The rebinning of the spectra to a scale linear in RV, which is needed as input for KOREL, was carried out with the help of the HEC35D program written by $\mathrm{PH}^{2}$. Relative fluxes for the new wavelength points are derived using INTEP (Hill 1982), which is a modification of the Hermite interpolation formula. It is possible to choose the initial and last wavelengths,

\footnotetext{
1 The freely distributed version from Dec. 2004.

2 The program HEC35D with User's Manual is available to interested users at ftp://astro.troja.mff.cuni.cz/hec/HEC35.
}

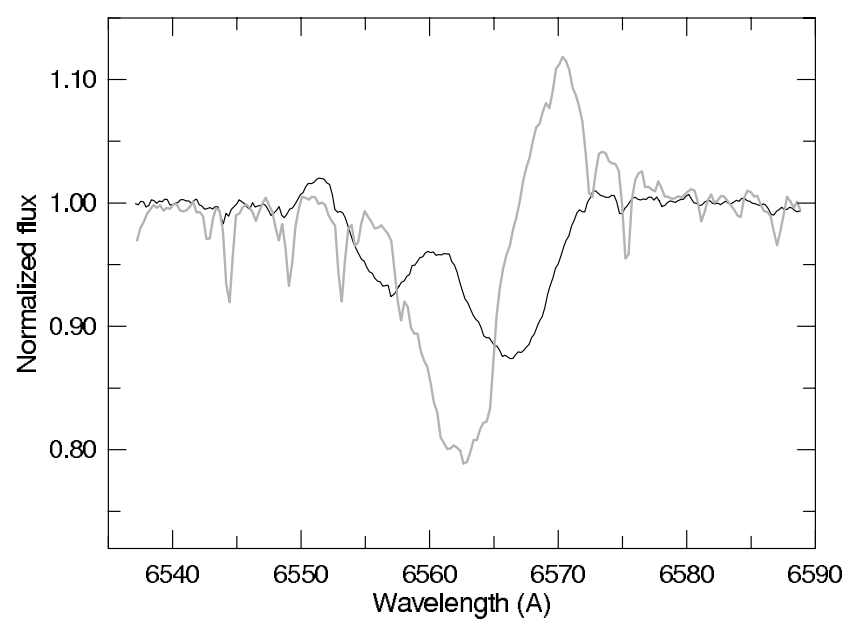

Fig. 4. Two examples of the observed $\mathrm{H} \alpha$ line profiles of LY Aur with emission at phases 0.726 (blue wing emission; black) and 0.449 (prominent red emission component; gray); the narrow features are the water vapour terrestrial absorptions.

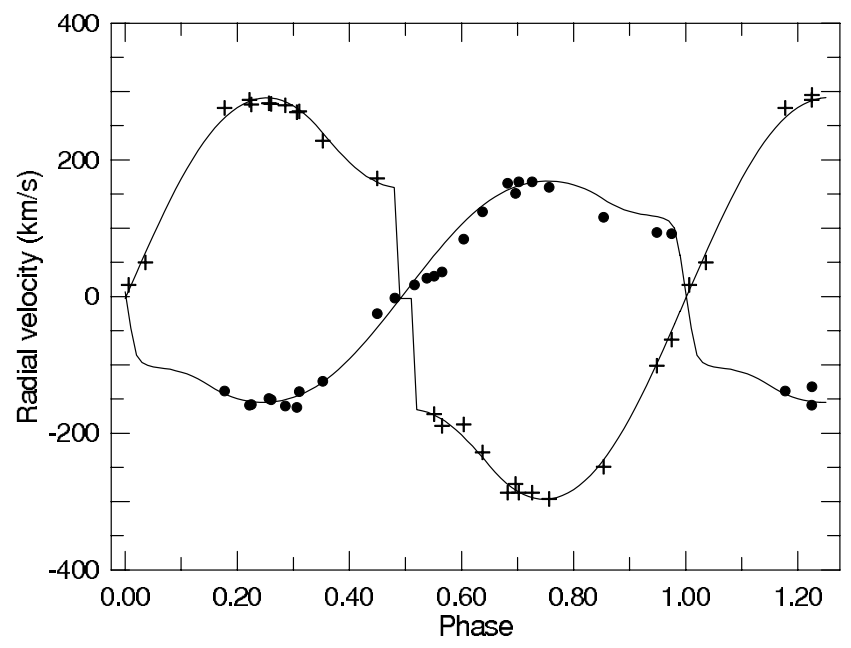

Fig. 5. Radial velocity curves of both components of LY Aur. The theoretical curves are drawn according to the $K_{1}, K_{2}$, and $V \gamma$ values of this paper.

and the program smoothly fills in the rebinned spectra with continuum values of 1.0 at both edges ${ }^{3}$.

To consider the variable quality of individual spectra, we measured their signal-to-noise ratios $(\mathrm{S} / \mathrm{N})$ in the line-free region 6640-6655 $\AA$ and assigned each spectrum a weight according to the formula

$w=\frac{(S / N)^{2}}{\left(S / N_{\text {mean }}\right)^{2}}$,

where $\mathrm{S} / \mathrm{N}_{\text {mean }}$ denotes the mean $\mathrm{S} / \mathrm{N}$ of all spectra. The spectra have an $\mathrm{S} / \mathrm{N}$ between 76 and 622 . We analysed the neighbourhood of the He I $6678 \AA$ line (6665-6695 $)$ ), using the RV step of $2.2 \AA \mathrm{mm}^{-1}$ per one bin.

We briefly recall that KOREL uses the observed spectra and derives both the orbital elements and the mean individual line profiles of the two binary components. Fixing the orbital elements found in the previous sections, and using the estimate of the long period of the AB system after Mason et al. (1998), we disentangled the observed spectra into three components.

3 This is necessary since KOREL requires that the number of the input data points be an integer power of 2 . 


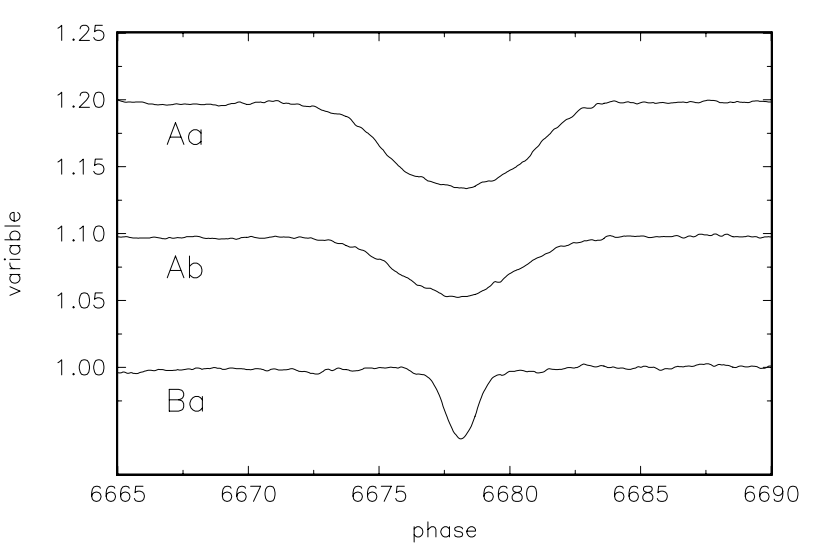

Fig. 6. Disentangled line profiles of the three components of the He I 6678 line. They are all normalized to the common continuum of the whole quadruple system, and their continua are shifted with a step of 0.1 for clarity.

We then kicked the KOREL in different directions from the previously found values to find out a free solution for more elements. We, however, kept the values of all three orbital periods and the epoch of the primary mid-eclipse of LY Aur fixed in all these trials, since their values are better constrained by the analyses presented in the previous sections.

The results obtained using KOREL are in a good agreement with the previous analyses and results for the visual component (Sect. 7). At the end, we also attempted to detect the secondary line of the visual component but in vein. This component could be of a later spectral type and its detection will probably require accumulation of a larger number of blue spectra.

The disentangled line profiles, normalized to the common continuum of all three (four) stars, are displayed in Fig. 6.

\section{Light-curve solution and parameters of the eclipsing binary}

Various light curves of LY Aur and solutions were hitherto published by several authors. Besides the data used by DLM, HIPPARCos measurements also exist; Perryman \& ESA (1997) list 66 points. An early light-curve solution was presented by Eaton (1978), who also used the UV measurements made by the satellite OAO2 (Heap 1973). He pointed out that the mass ratio $q=0.375$ published by $\mathrm{ABH}$ was probably incorrect. $\mathrm{Li}$ $\&$ Leung (1985) got the photometric ratio of 0.56 , in better accordance with the spectroscopic ratio 0.66 estimated by Popper (1982). DLM obtained a photometric $q=0.62$. However, it was found that a correlation between the mass ratio and the uncertain third-body contribution exists. The magnitude difference between LY Aur and its visual component was originally mentioned as 1. 5 according to Aitken \& Doolittle (1932). There are more recent measurements, but the disagreement with the third light fraction derived from light-curve solutions is still large; see Sect. 7.

Here, we present a new solution of a light curve in which the $U B V$ normal points of DLM and HIPPARCos data (transformed to the $V$ system according to Harmanec 1998) were combined. The solution was obtained with the MORO code (Drechsel et al. 1995), and the result is shown in Fig. 7. The photometric mass ratio is well defined between 0.56 and 0.57 and closely agrees with the spectroscopic value of 0.55 (see Sect. 4). The luminosity ratio $L_{2} / L_{1}$ is 0.51 , that is, the component contributions to the

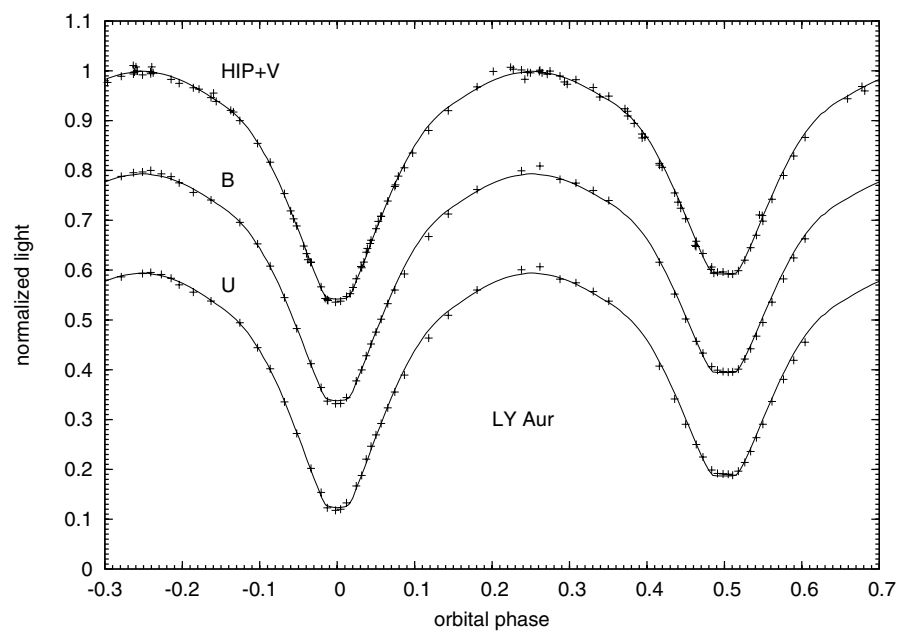

Fig. 7. Photometric solution of the $U B V$ normal points according to DLM combined with HIPPARCos $V$ data.

Table 4. Orbital parameters of LY Aur.

\begin{tabular}{lc}
\hline \hline Period [days] & $4.0024932(5)$ \\
Time of the periastron & $2439061.4646(16)$ \\
Eccentricity (assumed) & 0 \\
Semi-amplitude $K_{1}\left[\mathrm{~km} \mathrm{~s}^{-1}\right]$ & $161.9(1.6)$ \\
Semi-amplitude $K_{2}\left[\mathrm{~km} \mathrm{~s}^{-1}\right]$ & $294.3(2.1)$ \\
Systemic velocity $V \gamma_{1}\left[\mathrm{~km} \mathrm{~s}^{-1}\right]$ & 7 \\
Systemic velocity $V \gamma_{2}\left[\mathrm{~km} \mathrm{~s}^{-1}\right]$ & -3 \\
Semimajor axis $\left[R_{\odot}\right]$ & 36.1 \\
\hline
\end{tabular}

Notes. Only the spectra taken at phases closer than 0.11 to a quadrature were used.

Table 5. Light-curve solution and absolute parameters of LY Aur.

\begin{tabular}{lcc}
\hline \hline Inclination $[\mathrm{deg}]$ & 87.7 & \\
Mass ratio $M_{2} / M_{1}$ & 0.550 & \\
Luminosity ratio $(V)$ & 0.51 & \\
\hline & Primary & Secondary \\
\hline$T_{\text {eff }}[\mathrm{K}]$ & $31000^{a}$ & 31150 \\
Fract. radii & 0.448 & 0.351 \\
Masses $\left[M_{\odot}\right]$ & 25.5 & 14.0 \\
Radii $\left[R_{\odot}\right]$ & 16.1 & 12.6 \\
$\log L / L_{\odot}$ & 5.33 & 5.13 \\
$\log g[\mathrm{cgs}]$ & 3.425 & 3.378 \\
Rot. velocities $\left[\mathrm{km} \mathrm{s}^{-1}\right]$ & 194 & 152 \\
Synchronous rot. vel. $\left[\mathrm{km} \mathrm{s}^{-1}\right]$ & 204 & 160 \\
$M_{V}$ & -5.62 & -5.11 \\
\hline
\end{tabular}

Notes. ${ }^{(a)}$ Average of $T_{\text {observational }}$ and $T_{\text {spectroscopic }}$ for $\mathrm{O} 9 \mathrm{II}$ according to Martins et al. (2005).

total light (in $V$ ) are 0.56 (primary), 0.29 (secondary), and 0.15 (tertiary).

Using the RV data of Table 2, we calculated the orbital parameters as given in Table 4. Absolute parameters based on the new photometric solution are listed in Table 5. With an inclination of $i=87.7$, the masses come out as $M_{1}=25.5 M_{\odot}$ and $M_{2}=14.0 M_{\odot}$. The primary mass agrees well with the value for an 09 II star, as interpolated in the table of Martins et al. (2005), but the secondary mass appears rather low for its spectral type. 


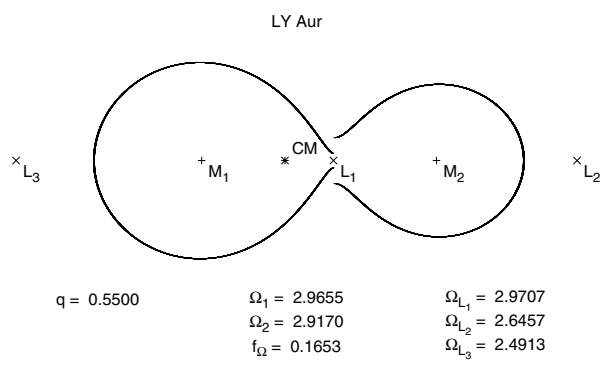

Fig. 8. Meridional intersection of Roche equipotentials; the primary fills its critical lobe, while the over-contact shape of the secondary is pretended by circumstellar matter. See text.

The new light-curve solution of the $U B V$ data of DLM combined with HIPPARCos $V$ data leads to a mass ratio, which now agrees with the spectroscopic result. If we would however restrict ourselves to the WD mode 3 for over-contact configurations, no solution with nearly equal temperatures $T_{\text {eff1 }}$ and $T_{\text {eff2 }}$, which would fit the different minimum depths, would be possible.

For this reason, we attempted further light-curve solutions using the WD mode 0 , where the secondary luminosity $L_{2}$ is not coupled to $T_{2}$ via the Planck law but can be freely adjusted. Mode 0 usually applies to detached and semi-detached systems. However, all trial runs led to inconsistent case of a binary, where the primary nearly completely fills its Roche lobe, while the secondary overfills its critical limit. Yet, it must be stressed that this configuration with a discontinuity of the surface potential at the inner Lagrangean point produces a very good theoretical representation of the observed light curve. It actually cannot be discerned at all from the one shown in Fig. 7, which has nearly exactly the same weighted sum of squared residuals as in the over-contact case (standard deviation 0.0060 versus 0.0059 intensity units). The shape of the Roche limits is shown in Fig. 8 as a meridional intersection.

It should be noted that this alternate solution was achieved for the spectroscopic mass ratio $q=0.55$ and that the main difference to the over-contact case is that $T_{\text {eff2 }}$ is now $100 \mathrm{~K}$ higher than $T_{\text {effl }}$, as suggested by the He line strengths. One can suspect that the apparent discontinuity of the surface at the contact point is only simulated by the presence of relatively dense circumstellar matter in the vicinity of the $L_{1}$ point. This material could emerge from a non-conservative mass transfer from the Rochelobe-filling primary towards the secondary star. This hypothesis is further supported by the following two observations:

First, the $\mathrm{O}-\mathrm{C}$ analysis shows that the orbital period has been generally decreasing over the past 40 years, which has been expected for the initial rapid mass transfer phase before the mass ratio reversal. On the other hand, the presence of circumstellar matter close to the surface of the two hot stars could also give rise to the observed $\mathrm{H} \alpha$ line emission.

\section{The third component}

In two papers where the light curve was solved, the amount of the third light contribution was given. Drechsel et al. (1989) found a value of $l_{3}=9.9 \%$ (in $V$ ) at a maximum quadrature phase, which corresponds to a difference of $2 \mathrm{~m} .40$. The Fourth Catalog of Interferometric Measurements ${ }^{4}$ lists the result by Maíz Apellániz (2010) of 1.87 at $\lambda 912 \mathrm{~nm}$ as the most precise magnitude difference. After transforming the Bessellian time

\footnotetext{
4 http://ad.usno.navy.mil/wds/int4.html
}

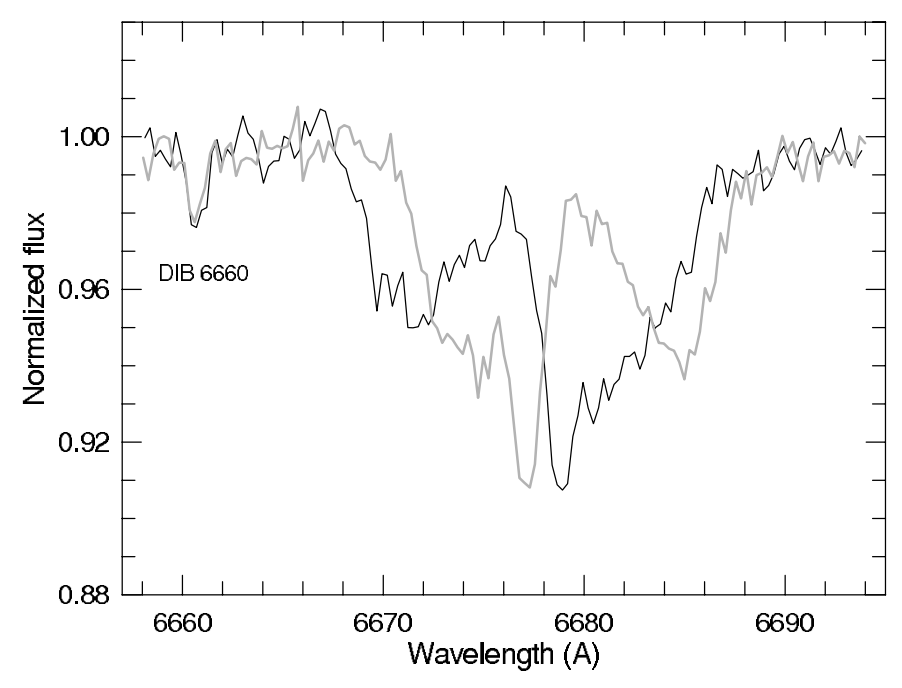

Fig. 9. Two examples of the He I 6678 spectral region of LY Aur with distinct lines of the visual companion at different positions (narrow features near the centre); spectra were taken at the eclipsing binary phases 0.696 (black) and 0.177 (grey).

to JD, the corresponding eclipsing binary phase is 0.219 , and the respective correction to the maximum magnitude is $0{ }^{\mathrm{m}} 01$. That is, the resulting difference is 1.88 . Since the visual component temperature is lower than that of the eclipsing binary components, a correction should be applied to estimate the right difference in the visual region: Using a correction of 0.03 , the resulting difference becomes $1 \mathrm{~m}$. 91 , and the corresponding ratio $L_{3} /\left(L_{1}+L_{2}+L_{3}\right)$ would be $14.7 \%$, which is considerably larger than the $L_{3}$ values resulting from various light-curve solutions. If we consider the HIPPARCOS (Perryman \& ESA 1997) measurement, which suggests a visual brightness difference of $2 \mathrm{~m} .19 \pm 0.08$, the corresponding luminosity ratio of $11.8 \pm 0.7 \%$ would be closer to the result of the light-curve solutions.

Contributions of the third component to the blended line profiles of Si IV 4089 and He I 4471 lines are apparent in two quadrature spectra, as shown in Fig. 1 of Popper (1982). In the ELODIE spectrum, third component features are also well detectable in the Si III 4553, 4568, and 4575 lines and in many O II lines (e.g., 4349 and 4415). For Si III and O II, contributions by the primary and secondary components are absent. However, no signature of the third body can be detected in He II blends with the uncertain exception of $\lambda 4686$. We compared the observed EWs of O II, C III, Si III, and He I to widths calculated from synthetic spectra by Lanz \& Hubeny (2007, assuming solar abundance and $\log g=4.0$ ) and got the best fit for a temperature of $T_{\text {eff }}=26000 \mathrm{~K}$ (Table 7). Therefore, the third body, the primary of the binary, might be of type B0.5; if it is a main sequence member, its contribution to the total light would be about $6 \%$. Considering the age of the eclipsing binary members is about $5 \mathrm{Myr}$, it might, however, be a giant, then the contribution would be $15 \%$. From the ratio of theoretical and observed widths, $l_{3}$ contributes $14 \%$ of the system total luminosity, which agrees with the direct measurements.

Stickland et al. (1994) gave the velocity of the third body as $+25 \mathrm{~km} \mathrm{~s}^{-1}$, claiming that the large difference from the binary systemic velocity $V \gamma$ of $+5 \mathrm{~km} \mathrm{~s}^{-1}$ might indicate that the third body is not physically bound to the LY Aur binary (a hardly supportable suggestion). Popper (1982) noted that the velocities measured near phase at 0.25 were $+24 \mathrm{~km} \mathrm{~s}^{-1}$ and near phase at 0.75 were $+5 \mathrm{~km} \mathrm{~s}^{-1}$. Our spectroscopic study can explain these findings: we found that the third-component lines do not 


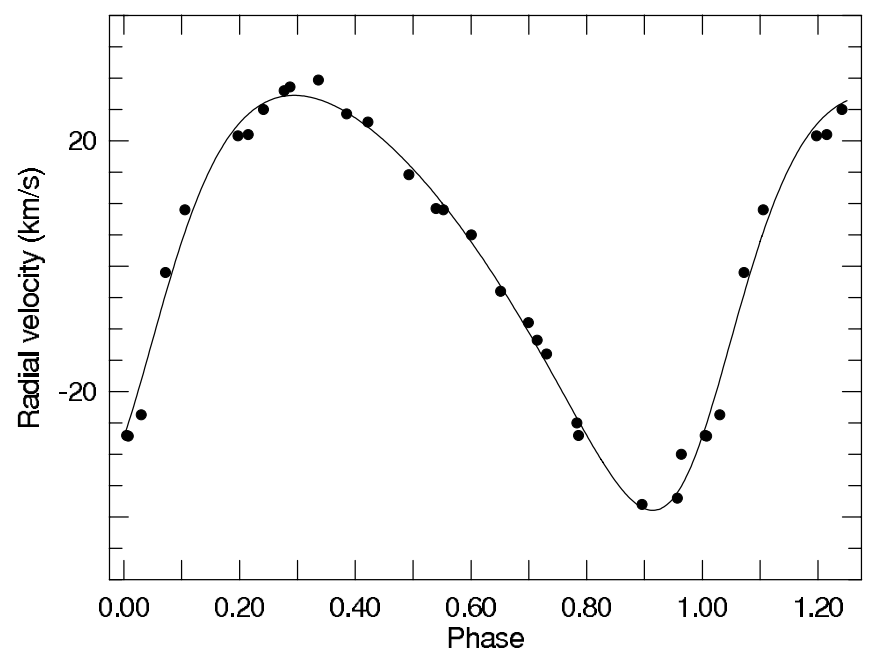

Fig. 10. Radial velocity curve of the visual companion of LY Aur. The theoretical curve is drawn according to the elements in Table 6.

Table 6. Elements of the orbit of the visual companion SB1 binary ADS 4072B.

\begin{tabular}{lc}
\hline \hline Period [days] & $20.4642(17)$ \\
Time of the periastron & $2454288.455(46)$ \\
Eccentricity & $0.246(17)$ \\
Longitude of the periastron [deg] & $229.9(5.2)$ \\
Semi-amplitude $K_{1}\left[\mathrm{~km} \mathrm{~s}^{-1}\right]$ & $33.0(8)$ \\
Systemic velocity $V \gamma\left[\mathrm{km} \mathrm{s}^{-1}\right]$ & $-0.6(5)$ \\
Rotational velocity $V \sin i\left[\mathrm{~km} \mathrm{~s}^{-1}\right]$ & $20(3)$ \\
Mass function $\left[M_{\odot}\right]$ & $0.0690(29)$ \\
$\mathrm{rms}\left[\mathrm{km} \mathrm{s}^{-1}\right]$ & 2.2 \\
\hline
\end{tabular}

Table 7. Equivalent widths of several lines of the visual component $(\AA)$.

\begin{tabular}{lccc}
\hline \hline Line & Tertiary & Theory $^{a}$ & Observation/Theory \\
\hline He I 4922 & 0.038 & 0.300 & 0.127 \\
He I 6678 & 0.073 & 0.463 & 0.158 \\
C III 4647 & 0.012 & 0.075 & 0.160 \\
O II 4349 & 0.015 & 0.112 & 0.134 \\
O II 4414 & 0.018 & 0.125 & 0.144 \\
O II 4649 & 0.017 & 0.141 & 0.121 \\
Si III 4552 & 0.019 & 0.144 & 0.132 \\
Si III 4568 & 0.020 & 0.117 & 0.171 \\
\hline
\end{tabular}

Notes. ${ }^{(a)}$ Calculated from synthetic spectra of Lanz \& Hubeny (2007) for $T_{\mathrm{eff}}=26000 \mathrm{~K}$ and $\log g=4.00$.

have fixed positions, their RVs vary within a range of nearly $70 \mathrm{~km} \mathrm{~s}^{-1}$, as seen in Fig. 9. Apparently, the third component is a binary itself. We succeeded in deriving the period and other elements of the SB1 orbit; see Table 6. The RV curve is shown in Fig. 10. Therefore, the system containing the eclipsing binary LY Aur is another case of a multiple system composed of two close binaries. These configurations are apparently common. Other examples are IU Aur (Drechsel et al. 1994), SZ Cam (Mayer et al. 2010), or QZ Car.

\section{Discussion and conclusions}

Although our results about parameters of both components of the eclipsing binary do not substantially differ from those known before, the value of the mass ratio is now more reliable. The small mass of the secondary found by previous investigators is confirmed. The behaviour of the $\mathrm{H} \alpha$ emission is now described in more detail.

We like to mention again the problem arising from the higher temperature of the secondary, as suggested by spectroscopic findings when compared to that resulting from the light curve solutions. The temperatures of the binary components should be reflected by their $\mathrm{He} \mathrm{I} / \mathrm{He}$ II $\mathrm{EW}$ ratios, but the difference is not as high as suggested by the classification of Sota et al. (2011). We prefer the classification O9 II+O9 III. To explain the small difference of the $\mathrm{He}$ I/He II EW ratios of both components we had to accept an uncommon solution of the light curve, which yields a $T_{\text {eff2 }}$ value that is slightly higher than $T_{\text {eff1 } 1}$. Its advantage is that it can also explain the origin of the interbinary matter, the occurrence of emission, and the period decrease.

We note that the secondary mass in another early-type contact binary, TU Mus, is also lower than what would correspond to its spectral type (e.g. Linder et al. 2007). Secondary masses smaller than those of single stars of the same type are expected, if the binary has passed or is currently in a phase of mass exchange. Penny et al. (2002) compared several semi-detached binaries and found that undermassive secondaries are commonly observed. The fill-out parameter of LY Aur is so small that the binary could be in an evolutionary phase that is comparable to interacting semi-detached systems. The light-curve solutions also always suggest a relatively low third light contribution of about $6 \%$, while the observed third light is larger. One can speculate that these contradictory results are connected with the presence of circumbinary matter.

According to Humphreys (1978), LY Aur is a member of the association Aur OB1 with a distance modulus of $10^{\mathrm{m}} 6$ (see also Stickland et al. 1994). Then the binary luminosity would be one magnitude lower than the expected value for the spectral types of its components. This is, however, unacceptable, since the luminosity cannot be so much in error as it follows from the derived temperatures and radii. The distance moduli as listed by Humphreys (1978) for the probable members of the association have a large range ( 9.7 to $11^{\mathrm{m}} .7$ ), and in the Aur OB1 field, there is also the association Aur OB2 with a modulus of 12.25 . The hypothetical membership in Aur OB1 therefore cannot really be used to derive the binary distance. According to our parameters, the distance modulus is $11 \mathrm{~m} .6$.

Our spectroscopic analysis unambiguously unveiled the third body in the system to be a close SB1 binary itself, for which we determined a precise RV curve and an accurate orbital solution. The system is, therefore, a new member of the small group of quadruple systems that consists of two close binaries.

Acknowledgements. We are grateful to Dr. A. Witt for allowing us to use his measurement of a minimum time and also to Drs. Li-ying Zhu and Ergang Zhao, who kindly communicated us their results in advance of publication. Reinald Lorenz is thanked for observations of the star at the Calar Alto Observatory. Drs. D. Korčáková, J. Nemravová, V. Votruba, M. Wolf, and P. Zasche helped in obtaining the spectra at the $2 \mathrm{~m}$ telescope in Ondřejov. We profitted from the use of the computerized bibliography maintained in the NASA/ADS system and the CDS in Strasbourg. P.M. and P.H. were supported by the grant P209/10/0715 of the Czech Science Foundation and also from the research program MSM0021620860.

\section{References}

Aitken, R. G., \& Doolittle, E. 1932, New general catalogue of double stars within $120^{\circ}$ of the North pole (Washington, D.C.: Carnegie Institution of Washington)

Andersen, J., Batten, A. H., \& Hilditch, R. W. 1974, A\&A, 31, 1

Conti, P. S., \& Alschuler, W. R. 1971, ApJ, 170, 325

Drechsel, H., Lorenz, R., \& Mayer, P. 1989, A\&A, 221, 49 
Drechsel, H., Haas, S., Lorenz, R., \& Mayer, P. 1994, A\&A, 284, 853 Drechsel, H., Haas, S., Lorenz, R., \& Gayler, S. 1995, A\&A, 294, 723 Eaton, J. A. 1978, Acta Astron., 28, 195

Hadrava, P. 2004, Publ. Astron. Inst. Acad. Sci. Czech Rep., 92, 15

Hall, D. S., \& Heiser, A. M. 1972, PASP, 84, 33

Harmanec, P. 1998, A\&A, 335, 173

Heap, S. R. 1973, ApJ, 186, 939

Hill, G. 1982, Publ. Dominion Astrophys. Obs. Victoria, 16, 67

Hubscher, J., Lehmann, P. B., Monninger, G., Steinbach, H.-M., \& Walter, F. 2010, IBVS, 5918, 1

Humphreys, R. M. 1978, ApJS, 38, 309

Kohoutek, L., \& Wehmeyer, R. 1997, Astronomische Abhandlungen der Hamburger Sternwarte, 11

Krajci, T. 2005, IBVS, 5592, 1

Landolt, A. U., \& Blondeau, K. L. 1972, PASP, 84, 394

Lanz, T., \& Hubeny, I. 2003, ApJS, 146, 417

Lanz, T., \& Hubeny, I. 2007, ApJS, 169, 83

Li, Y.-F., \& Leung, K.-C. 1985, ApJ, 298, 345

Linder, N., Rauw, G., Sana, H., De Becker, M., \& Gosset, E. 2007, A\&A, 474, 193

Maíz Apellániz, J. 2010, A\&A, 518, A1

Martins, F., Schaerer, D., \& Hillier, D. J. 2005, A\&A, 436, 1049
Mason, B. D., Gies, D. R., Hartkopf, W. I., et al. 1998, AJ, 115, 821

Mayer, P. 1968, PASP, 80, 81

Mayer, P. 1980, IBVS, 1724, 1

Mayer, P., \& Horák, T. B. 1971, Bull. Astron. Inst. Czech., 22, 327

Mayer, P., Wolf, M., Niarchos, P. G., et al. 2006, Ap\&SS, 304, 39

Mayer, P., Drechsel, H., Kubát, J., \& Sllechta, M. 2010, A\&A, 524, A1

Moultaka, J., Ilovaisky, S. A., Prugniel, P., \& Soubiran, C. 2004, PASP, 116 , 693

Penny, L. R., Gies, D. R., Wise, J. H., Stickland, D. J., \& Lloyd, C. 2002, ApJ, 575,1050

Perryman, M. A. C., \& ESA 1997, The Hipparcos and Tycho catalogues, Astrometric and photometric star catalogues derived from the ESA Hipparcos Space Astrometry Mission (Noordwijk, Netherlands: ESA Publications Division), ESA SP Ser., 1200

Popper, D. M. 1982, ApJ, 262, 641

Sota, A., Maíz Apellániz, J., Walborn, N. R., et al. 2011, ApJS, 193, 24

Stickland, D. J., Koch, R. H., Pachoulakis, I., \& Pfeiffer, R. J. 1994, The Observatory, 114, 107

Thaller, M. L. 1997, ApJ, 487, 380

Thaller, M., \& Gies, D. 1996, in Rev. Mex. Astron. Astrofis. Conf. Ser. 5, eds. V. Niemela, N. Morrell, P. Pismis, \& S. Torres-Peimbert, 117

Wilson, R. E., \& Devinney, E. J. 1971, ApJ, 166, 605 